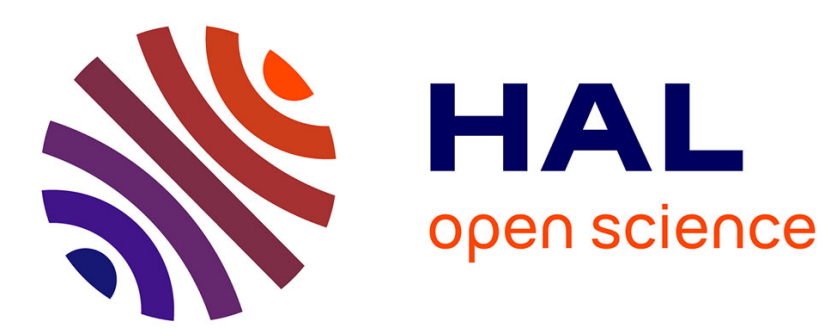

\title{
Diagnostic positif et étiologique des démences frontotemporales
}

\author{
T. Lebouvier, Maxime Bertoux, M. Leroy, F. Lebert, V. Deramecourt, F.
} Pasquier

\section{- To cite this version:}

T. Lebouvier, Maxime Bertoux, M. Leroy, F. Lebert, V. Deramecourt, et al.. Diagnostic positif et étiologique des démences frontotemporales. Pratique Neurologique - FMC, 2019, 10, pp.101 - 111. 10.1016/j.praneu.2019.02.012 . hal-03486518

\section{HAL Id: hal-03486518 \\ https://hal.science/hal-03486518}

Submitted on 20 Dec 2021

HAL is a multi-disciplinary open access archive for the deposit and dissemination of scientific research documents, whether they are published or not. The documents may come from teaching and research institutions in France or abroad, or from public or private research centers.
L'archive ouverte pluridisciplinaire HAL, est destinée au dépôt et à la diffusion de documents scientifiques de niveau recherche, publiés ou non, émanant des établissements d'enseignement et de recherche français ou étrangers, des laboratoires publics ou privés.

\section{다)(1) $(5$}

Distributed under a Creative Commons Attribution - NonCommercial| 4.0 International 
Version of Record: https://www.sciencedirect.com/science/article/pii/S1878776219300408

Manuscript_7c3777771bcc9adc085a298d2297e2f5

\section{Diagnostic positif et étiologique des démences frontotemporales}

\section{Positive and etiological diagnosis of frontotemporal dementias}

Thibaud Lebouvier ${ }^{1 *}$, Maxime Bertoux ${ }^{2}$, Mélanie Leroy ${ }^{2}$, Florence Lebert ${ }^{3}$, Vincent Deramecourt $^{1}$ et Florence Pasquier ${ }^{2 *}$

${ }^{1}$ Univ.Lille, Inserm U1172, CHU Lille, DISTALZ, F-59000 Lille, France

${ }^{2}$ Univ.Lille, Inserm U1171, CHU Lille, DISTALZ, F-59000 Lille, France

${ }^{3}$ CHU Lille, DISTALZ, F-59000 Lille, France

*Correspondance :

Thibaud Lebouvier - CHU de Lille - Clinique Neurologique, CMRR - 59037 Lille Cedex

Tél. 03.20.44.60.21

thibaud.lebouvier@chru-lille.fr 


\title{
Résumé
}

Le diagnostic positif et étiologique de la variante comportementale des dégénérescences lobaires frontotemporales (DLFT), également appelé démence frontotemporale (DFTc), reste une gageure en 2019. Le diagnostic différentiel entre DFTc et troubles psychiatriques peut être aidé par l'utilisation stricte des critères diagnostiques du DSM5 et quelques astuces sémiologiques. Le diagnostic différentiel avec les présentations exécutives/comportementales de maladie d'Alzheimer (MA) est également difficile car les troubles de la mémoire épisodiques sont fréquents dans la DFTc. En outre les biomarqueurs de MA du liquide cérébrospinal peuvent être positifs en cas de co-pathologie ou faussement positifs dans certaines DFTc. Enfin le diagnostic étiologique des présentations cliniques de DFTc, qui peuvent correspondre à de multiples pathologies appartenant ou non au spectre des DLFT, est particulièrement difficile. Du fait de leur fréquence, la recherche de mutations $G R N$ ou C9orf72 devrait être proposée dans tous les cas de DFTc ; la recherche de mutations plus rares peut être réservée aux cas précoces ou familiaux. Dans les cas sporadiques, certains phénotypes clinico-radiologiques associés aux DFTc doivent être bien connus car prédictifs de la pathologie sous-jacente, comme l'association à une pathologie motoneuronale dans les DFTc liées à une DLFT-TDP de type B. L'avenir verra le développement de marqueurs cliniques, biologiques ou d'imagerie pour améliorer les corrélations clinicopathologiques et ouvrir la voie aux essais thérapeutiques de traitements neuroprotecteurs.

Mots-clefs : Dégénérescence lobaire fronto-temporale, Démence frontotemporale, Maladie d'Alzheimer, Corrélations clinico-pathologiques

\begin{abstract}
Positive and etiological diagnosis of the behavioral variant of frontotemporal lobar degeneration (FTLD), also known as frontotemporal dementia (bvFTD), is still a challenge in 2019. The differential diagnosis with psychiatric disorders can be better achieved with clinical experience combined with a strict use of DSM5 criteria. The executive/behavioral variant of Alzheimer's disease is also a difficult differential diagnosis, since episodic memory impairment is frequent in bvFTD. In that matter, the interpretation of positive Alzheimer's disease cerebrospinal fluid biomarkers should be cautious in order not to rule out an association of pathologies or a false-positivity. Last, the bvFTD clinical phenotype is underlain by multiple possible pathologies belonging or not to the FTLD spectrum. Due to their prevalence, screening for mutations in GRN and C9orf72 genes should be offered to all bvFTD patients. Rarer mutations should be looked for in early onset and/or familial cases. In sporadic bvFTD, certain clinical and neuroimaging profiles are well correlated to the
\end{abstract}


underlying pathology, such as bvFTD with motor neuron disease and FTLD-TDP type B pathology. The future will see the advent of clinical, neuroimaging and biological markers to increase clinicopathological correlations and facilitate clinical trials of disease-modifying drugs.

Keywords: Frontotemporal lobar degeneration, Frontotemporal dementia, Alzheimer's disease, Clinicopathological correlations 


\section{Abréviations}

$\begin{array}{ll}\text { APP } & \text { Aphasie primaire progressive } \\ \text { APPnf } & \text { APP de type agrammatique non fluente } \\ \text { APPs } & \text { APP de type asémantique (=démence sémantique) } \\ \text { DCB } & \text { Dégénérescence cortico-basale } \\ \text { DFTc } & \text { Variante comportementale de DLFT (=démence frontotemporale) } \\ \text { DLFT } & \text { Dégénérescence lobaire frontotemporale } \\ \text { FUS } & \text { fused-in-sarcoma } \\ \text { MA } & \text { Maladie d'Alzheimer } \\ \text { PSP } & \text { Paralysie supranucléaire progressive } \\ \text { SLA } & \text { Sclérose latérale amyotrophique } \\ \text { TDP } & \text { TAR DNA-binding protein } 43\end{array}$

\section{Introduction}

Le renouvellement des critères diagnostiques de maladies neurodégénératives traduit le dynamisme de notre discipline. Dans un effort constant pour améliorer les corrélations entre le phénotype clinique et la pathologie sous-jacente, toutes les grandes maladies neurocognitives ont vu leurs critères diagnostiques actualisés au cours des dix dernières années [1-5]. Avec le développement de nouvelles molécules ciblant directement le processus pathologique, il devient capital de pouvoir prédire de façon fiable, du vivant du patient, la nature de la pathologie neurodégénérative sous-jacente. L'enjeu est dès aujourd'hui de pouvoir inclure les patients dans des essais thérapeutiques, et de se préparer à l'arrivée espérée de thérapeutiques ciblées efficaces.

C'est dans le domaine des dégénérescences lobaires frontotemporales (DLFT) qu'il reste le plus de progrès à effectuer. Les DLFT constituent un groupe de pathologies très diverses caractérisées par une dégénérescence relativement sélective des lobes frontaux et/ou temporaux. Elles se manifestent préférentiellement mais non exclusivement par trois syndromes cliniques (ou «variantes») : la variante comportementale (DFTc) d'une part, également appelée démence frontotemporale, est la manifestation clinique la plus fréquente des DLFT ; les deux variantes de langage d'autre part sont les aphasies progressives primaires (APP) de type sémantique (APPs), ou démence sémantique, et les APP de type agrammatique non fluente (APPnf), qui se distinguent des APP logopéniques le plus souvent associées à une forme focale de MA [6]. Ce démembrement des APP a eu le mérite d'améliorer les corrélations clinico-pathologiques [7]. 
Ainsi à l'heure actuelle, c'est le phénotype clinique de DFTc qui demeure la terra incognita des maladies neurodégénératives. Une présentation de DFTc peut correspondre à près de 14 pathologies différentes, incluant la maladie d'Alzheimer dans plus de 10\% des cas [8] ! En outre les critères de DFTc peuvent être remplis par de nombreux troubles psychiatriques [9]. Et si les progrès de la biologie moléculaire permettent de poser un diagnostic étiologique dans les principales formes génétiques de DLFT, les formes sporadiques de DFTc demeurent un défi en 2018.

L'objectif de ce travail est d'aider le clinicien à affiner son diagnostic étiologique face à un patient présentant une DFTc. Après un survol nécessaire de l'hétérogénéité clinique, pathologique et génétique du spectre des DLFT, deux enjeux majeurs du diagnostic de DFTc seront abordés : (1) le diagnostic différentiel entre DLFT, MA et trouble psychiatrique devant une présentation de DFTc ; (2) le diagnostic étiologique des DFTc, c'est-à-dire la prédiction de la pathologie sous-jacente.

\section{Hétérogénéité clinique, pathologique et génétique des DLFT}

\section{Hétérogénéité clinique}

Les DLFT sont la deuxième cause de démence du sujet jeune (c'est-à-dire survenant avant l'âge de 65 ans). La plupart des DLFT se manifestent cliniquement par l'une des trois variantes syndromiques évoquées ci-dessus : la variante comportementale (DFTc), la plus fréquente, ou l'une des deux variantes de langage (APPs et APPnf). En outre, les patients souffrant de DLFT peuvent développer un syndrome parkinsonien atypique ou une maladie du motoneurone au cours de la maladie, précocement ou tardivement, ce qui étend le spectre des manifestations cliniques possibles à la sclérose latérale amyotrophique (SLA), au syndrome de paralysie supranucléaire progressive (PSP) et au syndrome cortico-basal [10]. Ainsi, un patient débutant la maladie par une APPnf ou des troubles du comportement remplissant les critères de DFTc peut secondairement développer des signes de paralysie supranucléaire progressive ou une SLA.

\section{Hétérogénéité pathologique}

Comme la plupart des maladies neurodégénératives, les DLFT sont caractérisées d'un point de vue histopathologique par des inclusions neuronales ou neuro-gliales, qui reflètent une accumulation intracellulaire anormale de protéines spécifiques. Dans l'immense majorité des DLFT, le composant protéique principal des inclusions est l'une des trois protéines majeures permettant de classer la majorité des DLFT en trois classes moléculaires : la protéine Tau, codée par le gène $M A P T$, s'agrège dans les DLFT-Tau; la protéine TAR DNA-binding 
protein 43 (TDP) est le composant principal des inclusions dans les DLFT-TDP; enfin la protéine fused-in-sarcoma (FUS) s'agrège dans les DLFT-FUS, largement minoritaires. Des formes beaucoup plus rares de DLFT sont caractérisées par des inclusions ubiquitinylées nonTDP et non-FUS ou par l'absence de lésion histologique distinctive [11] (figure 1).

Les DLFT-TDP sont majoritaires (plus de 50\% des cas) et sont subdivisées en quatre soustypes histologiques, en fonction de la localisation et de la morphologie des inclusions (type A, B, C ou D). Les DLFT-Tau représentent environ $40 \%$ des cas et sont divisées en deux ou trois groupes, selon si la protéine Tau agrégée présente trois (Tau 3R) ou quatre (Tau 4R) domaines répétés de fixation aux microtubules, ou un mélange des deux. Les sous-types histologiques principaux des DLFT-Tau comprennent la maladie de Pick, qui est l'unique représentante des DLFT-Tau 3R; et la PSP, la dégénérescence corticobasale (DCB) et la maladie à grains argyrophiles, qui sont des DLFT-Tau 4R. PSP et DCB désignent ici les entités pathologiques caractérisées par des inclusions neurogliales spécifiques de protéine Tau $4 \mathrm{R}$, et non les syndromes cliniques emblématiques qui ne sont pas toujours présents [12].

Certains phénotypes cliniques sont plus volontiers associés à l'un des sous-types histologiques de DLFT : les APPs sont par exemple assez spécifiques aux DLFT TDP de type C. Mais la DFTc est une présentation clinique possible de l'ensemble des DLFT.

\section{Hétérogénéité génétique}

$\mathrm{Au}$ sein des MA et apparentées, les DLFT sont celles qui possèdent le déterminisme génétique le plus important. Une histoire familiale est retrouvée dans 20 à $50 \%$ des cas de DLFT. Qu'elle soit présente ou non, on estime que 10 à 20\% des DLFT sont causées par des mutations autosomales dominantes sur l'un des trois gènes suivants : MAPT, GRN (qui code pour la protéine progranuline, qu'on peut doser dans le sérum) et C9orf72 (qui code pour la protéine C9orf72). D'autres gènes sont beaucoup plus rarement impliqués : $C H M P 2 B, V C P$, SQSTM1, TARDP (qui code la protéine TDP $\square 43$ ) et $T B K 1$. Ajoutant à la complexité des DLFT, les corrélations entre génotype et phénotype sont faibles, avec une grande variabilité de l'expression des mutations au sein d'une même famille. En revanche, le génotype détermine la pathologie sous-jacente : les mutations $G R N$ sont constamment associées à une DLFT-TDP de type A ; les mutations C9orf72 à une pathologie DLFT-TDP de type B ou A ; les exceptionnelle mutations $V C P$ à une pathologie DLFT-TDP de type $\mathrm{D}$; et les mutations MAPT à une DLFT-Tau 3R, 4R ou mixte (figure 1) [13].

\section{Diagnostic positif des DFTc}


Le statut de diagnostic syndromique ou étiologique de la DFTc fait l'objet d'une certaine ambigüité. Le phénotype clinique de DFTc est partagé par des pathologies très diverses, qui appartiennent au spectre des DLFT ou se situent en dehors. Dans ce sens, il s'agit surtout d'un diagnostic syndromique qui ne permet pas de préjuger de la pathologie sous-jacente. Pour autant le syndrome de DFTc a été conçu dès l'origine pour décrire la présentation clinique principale des pathologies du groupe des DLFT, par opposition aux autres affections neuropsychiatriques ou neurodégénératives. Il s'agit dans ce sens d'un diagnostic étiologique. C'est à l'aune de leur ambition de diagnostic différentiel avec les pathologies non-DLFT que l'on peut critiquer ou apporter des précisions aux critères actuels de DFTc. L'objectif est de mieux distinguer les variantes comportementales de DLFT des autres étiologies, notamment des formes comportementales de MA et des troubles psychiatriques.

\section{Critères diagnostiques actuels}

Proposés par Rascovsky en 2011 [14], les critères diagnostiques de DFTc requièrent un déclin progressif des performance cognitives et l'apparition insidieuse et persistante de troubles du comportement spécifiques, regroupés en 5 critères (tableau 1). Ces troubles du comportement peuvent être associés à un profil neuropsychologique particulier, impliquant un déficit des fonctions exécutives et une préservation relative de la mémoire épisodique et des fonctions visuo-spatiales (sixième critère). Trois critères sont nécessaires pour porter un diagnostic de DFTc possible. Le diagnostic devient probable quand ils sont accompagnés à la fois d'un déclin fonctionnel dans la vie quotidienne et d'une atteinte des régions frontales ou temporales antérieures révélée par l'imagerie anatomique ou fonctionnelle (tableau 1).

Le diagnostic clinique de DFTc nécessite une anamnèse détaillée menée avec les proches et combinée à l'observation du patient au cours de la consultation. Les comportements pathologiques doivent avoir été observés dans les trois dernières années et tous doivent constituer une rupture avec le comportement ou la personnalité antérieure du patient. En l'absence de mesures précises ou objectives du comportement, le diagnostic clinique repose donc sur l'habileté des cliniciens à observer les malades et à écouter le discours des proches du patient. Si objectiver une diminution des activités sociales ne pose généralement pas de difficulté, notamment chez des patients encore en activité professionnelle ou parents d'adolescents, juger de la présence d'un manque d'empathie ou d'une perte des convenances sociales demande de s'adapter au contexte culturel et socio-économique. Même en s'aidant d'échelles complémentaires, la validation de ces critères implique un certain degré de subjectivité, une critique fréquemment formulée. 
Différents outils peuvent aider au recueil et à la quantification des troubles du comportement. En France, trois outils sont d'utilisation fréquente : l'Echelle de Dysfonctionnement Frontal (EDF) [15], catégorielle, utile au diagnostic au stade débutant (MMS>18); l'échelle DAPHNE (pour Désinhibition, Apathie, Persévérations, Hyperoralité, Négligence \& perte d'Empathie) qui reprend les critères de Rascovsky en leur ajoutant la négligence physique et permet de quantifier la sévérité des troubles du comportement [16] ; l'Inventaire du Syndrome Dysexécutif Comportemental qui permet également une quantification mais n'est pas spécifique aux DLFT [17].

Parmi les critiques faites à ces nouveaux critères, celles dirigées spécifiquement contre le critère neuropsychologique de «relative préservation de la mémoire épisodique » viennent à la fois appuyer cette notion de subjectivité (qu'est-ce qu'une préservation « relative»?) tout en opposant un faisceau de données neuropsychologiques contredisant clairement la préservation des fonctions mnésiques dans les DLFT confirmées histologiquement. Cette notion est particulièrement importante au moment d'aborder le diagnostic différentiel avec la MA.

\section{DFTc amnésiques et formes dysexécutives/comportementales de MA}

Malgré les premières descriptions clinicopathologiques d'Arnold Pick qui montraient déjà une amnésie sévère chez certains patients, l'atteinte de la mémoire épisodique a longtemps été sous-estimée dans les DLFT [18]. Or 30 à 60\% des patients remplissant les critères de DFTc présentent un déficit mnésique authentique, c'est à dire un trouble de l'encodage, du stockage et de la consolidation mnésique, bien au-delà du trouble de récupération classiquement attendu dans le cadre d'une atteinte exécutive. L'analyse des performances en mémoire de patients DFTc ayant des biomarqueurs de MA normaux montre une distribution bimodale, c'est à dire l'existence de deux populations distinctes situées de part et d'autre du seuil de normalité au test de mémoire [19]. L'existence d'une variante amnésique des DLFT comportementales qui concernerait environ $50 \%$ des patients semble dès lors établie.

Ainsi le diagnostic de DLFT ne doit pas être écarté si l'amnésie est sévère et/ou inaugurale, même en cas de profil «hippocampique », et l'analyse de la mémoire ne permet pas de discriminer efficacement DLFT et MA devant une présentation comportementale. C'est souligner l'importance de l'anamnèse dans l'évaluation des patients présentant une plainte même mnésique, incluant la recherche systématique de modifications du comportement. L'évaluation neuropsychologique conserve un intérêt majeur, notamment par l'évaluation des fonctions postérieures, qui sont relativement préservées dans les DLFT. L'évaluation des fonctions de cognition sociale ont en outre montré un très bon pouvoir discriminant entre 
DLFT comportementale et MA ; elle est particulièrement indiquée lorsque la présence d'une amnésie complique la distinction clinique [20] (tableau 1).

Cependant, rarement, une présentation apathique de DFTc peut révéler une MA. En d'autres termes, la DFTc peut constituer l'un des syndromes focaux révélateurs d'une MA atypique. Quelques indices permettent d'évoquer cliniquement une MA. En ce qui concerne le comportement, outre que l'apathie est le symptôme dominant, les critères de comportement stéréotypé/ritualisé ou d'hyperoralité sont rarement remplis. Sur le plan cognitif, ces patients présentent une atteinte sévère des fonctions exécutives, quasi-constante et plus sévère que celle observée dans les DLFT comportementales, et des troubles de la mémoire épisodique similaires à ceux de la MA. Sur le plan anatomique enfin, l'atrophie et l'hypométabolisme prédominent généralement dans le cortex temporal et cingulaire postérieur et le precuneus, comme dans la MA typique. L'atteinte des régions frontales est modérée, plus marquée que dans la MA typique mais bien moins importante que dans la DFTc. En somme, si ces patients peuvent remplir les critères de DFTc possible, ils remplissent plus rarement ceux de DFTc probable en l'absence de patron d'atrophie ou d'hypométabolisme évocateur de DLFT [8,21]. Ainsi, face à un patient amnésique remplissant les critères de DFTc, le clinicien doit garder en tête que les formes amnésiques de DLFT comportementale sont bien plus fréquentes que les formes dysexécutives/comportementales de MA, surtout si le patient présente un trouble du comportement alimentaires, des persévérations et une altération de la cognition sociale, et s'il remplit les critères de DFTc probable.

Les biomarqueurs de MA sont évidemment ici une aide précieuse au diagnostic : chaque fois que possible, devant un phénotype de DFTc, il convient de s'assurer de la normalité des biomarqueurs de MA (Tau, phospho-Tau, peptide $A \beta_{42}$ et ratio $A \beta_{42 / 40}$ ) dans le liquide cérébrospinal. Cependant si la positivité des biomarqueurs doit faire évoquer un variant comportemental de MA, elle n'est pas pathognomonique. Le diagnostic de DLFT ne doit pas être écarté sur des résultats marginalement positifs sur un ou plusieurs marqueurs de MA, possible dans les DLFT. En outre, et c'est surtout vrai chez le sujet âgé et chez les porteurs de l'allèle ApoE4, il faut savoir évoquer une DLFT associée à une MA devant des biomarqueurs de MA positifs et un phénotype clinique et en neuroimagerie caractéristique de DLFT, l'association des deux pathologies surtout chez des personnes âgées n'étant pas exceptionnelle [7].

\section{Syndromes de DFTc liés à un trouble psychiatrique}

L'une des difficultés du diagnostic de DFTc est que les troubles du comportement qui forment les critères du diagnostic appartiennent habituellement au domaine de la psychiatrie. Ainsi les 
critères de DFTc possible peuvent être validés dans un certain nombre de troubles psychiatriques. Le contexte (c'est-à-dire l'âge de début, l'ancienneté des troubles) aide le plus souvent à écarter une DFTc, dont les critères diagnostiques prévoient que les altérations comportementales ne doivent pas être mieux expliquées par un diagnostic psychiatrique [14] (tableau 1). Néanmoins le diagnostic différentiel avec un trouble psychiatrique de début tardif peut être particulièrement délicat.

Quelques astuces sémiologiques peuvent d'abord aider à orienter le clinicien. Si un épisode dépressif majeur peut se manifester par une apathie et des troubles attentionnels et exécutifs donnant le change avec une DFTc, la tristesse de l'humeur et les idées de culpabilité ou l'autodépréciation et l'idéation suicidaire sont absentes dans la DFTc ; à l'inverse l'atteinte de cognition sociale plaide en faveur de la DFTc devant une présentation pseudo-dépressive. L'état maniaque du trouble bipolaire partage des caractéristiques communes avec la désinhibition comportementale. Mais l'élation de l'humeur, les idées de grandeur ou d'invulnérabilité et l'insomnie sont rares dans la DFTc. A l'inverse négligence physique et perte d'empathie, deux des quatres symptômes de l'EDF, sont peu fréquents dans la bipolarité. Le trouble obsessionnel compulsif partage avec la DFTc le comportement persévératif ; néanmoins les compulsions d'origine psychiatrique sont toujours accompagnées d'un trouble obsessionnel et anxieux. Enfin apathie, perte d'empathie et troubles de cognition sociale sont retrouvés dans la schizophrénie dont le début peut être tardif, mais les symptômes positifs caractéristiques de la sémiologie psychiatriques (délire systématisé, hallucinations complexes) sont habituellement absents [9,22]. D'une manière générale, l'utilisation stricte des critères du DSM-5 permet souvent de repérer ces atypies devant une présentation pseudo-psychiatrique [9].

L'analyse semi-quantitative des troubles du comportement est une autre clef du diagnostic différentiel. Si nombre de troubles psychiatriques peuvent présenter certains critères de DFTc, l'intensité de la symptomatologie diffère; par une approche semi-quantitative des échelles comme DAPHNE permettent de distinguer les DLFT de la maladie bipolaire [16]. L'examen neurologique permet occasionnellement de mettre en évidence certains signes cliniques (syndrome parkinsonien non expliqués par les neuroleptiques, syndrome pyramidal, amyotrophie ou fasciculations) qui contribueront fortement au diagnostic différentiel [23]. Enfin la mise en évidence d'une histoire familiale de troubles cognitifs et comportementaux est une information majeure, la sémiologie psychiatrique étant particulièrement fréquente dans les formes génétiques de DLFT [9].

L'imagerie cérébrale (IRM encéphalique souvent complétée par une TEP-FDG) est indiquée dans toutes les troubles psychiatriques de révélation tardive posant le problème du diagnostic 
différentiel avec une DLFT, a fortiori s'il existe des atypies et si elles remplissent les critères de DFTc. Si la mise en évidence d'une atrophie ou d'un hypométabolisme frontotemporal franc aidera pour une orientation neurologique, le clinicien doit rester conscient que l'imagerie peut être normale dans les DLFT débutantes et qu'un hypométabolisme frontal modéré bilatéral est possible dans certaines troubles psychiatriques (trouble bipolaire, épisode dépressif majeur) [22].

Le diagnostic doit enfin pouvoir être remis en cause avec le suivi évolutif [24]. Des DLFT certaines, notamment liées à des mutations C9orf72, présentent une longue histoire de trouble bipolaire, schizophrénie ou trouble du spectre autistique avant de développer un phénotype plus complet de DFTc [9]. A l'inverse certains patients désignés sous le terme de «phénocopies » remplissent les critères de DFTc possible, incluant un syndrome dysexécutif modéré, mais leurs symptômes ne progressent pas sur plusieurs décennies et ces patients n'ont pas de lésion neurodégénérative à l'autopsie [25]. En majorité des hommes, ils se distinguent des DLFT par le respect relatif de l'autonomie et l'absence d'anomalie en neuroimagerie. Rares, ces «phénocopies » représentent un défi diagnostique; il pourrait s'agir de la révélation tardive de troubles psychiatriques divers [26]. Ainsi, il faut rester prudent avant de poser un diagnostic lourd de conséquences de maladie neurodégénérative devant une DFTc possible.

\section{Diagnostic étiologique des DFTc}

Une fois le diagnostic syndromique de DFTc établi, et les diagnostics différentiels non-DLFT raisonnablement écartés, la gageure est de porter un diagnostic étiologique. Les progrès de la génétique ont précédé ceux de la clinique dans cette entreprise. Cependant, même dans les formes sporadiques, des nuances cliniques peuvent aider au diagnostic étiologique. En l'absence de mutation identifiée, ce dernier restera un diagnostic de probabilité jusqu'à une éventuelle vérification post mortem.

\section{Place de l'analyse génétique}

La stratégie d'analyse, réalisée dans le respect des procédures de conseil génétique, est aujourd'hui bien codifiée [13]. Elle passe par un arbre généalogique permettant de visualiser les antécédents familiaux et de préciser le mode de transmission du phénotype. Les mutations GRN et C9orf72 expliquant la moitié des causes de DFTc familiales et jusqu'à 10\% des DFTc sporadiques, les analyses génétiques sont indiquées, après information et consentement, chez tous les patients, dans les formes familiales comme sporadiques. 
Le protocole débute par le dosage de la progranuline plasmatique, dont la diminution doit faire rechercher une mutation $G R N$. Si la progranulinémie est normale, une recherche d'expansion pathologique sur le gène C9orf72 est effectuée dans la foulée. La recherche de mutation $M A P T$ est à l'heure actuelle réservée aux formes familiales à début précoce (avant 55 ans), surtout en cas de phénotype évocateur de DLFT-Tau (syndrome corticobasal ou de PSP). Rarement une mutation VCP est envisagée en cas d'association de la DFTc à une myopathie à inclusions et/ou à une maladie de Paget. En cas de forme familiale sans étiologie retrouvée à l'issue de ces premières analyses, ou en cas de forme sporadique de début très précoce, des mutations peuvent être recherchées sur un panel de gènes.

Le phénotype clinique des DLFT liées aux mutations $G R N$ et C9orf72 est aujourd'hui bien connu. Les DLFT-TDP de type A liées à une mutation GRN sont caractérisées par la grande variabilité du phénotype clinique, y compris au sein de la même famille. Elles sont révélées par un tableau de DFTc dans $60 \%$ des cas, mais peuvent également se manifester par une APPnf ou un syndrome cortico-basal; la fréquence des hallucinations visuelles peut parfois donner le change avec une démence à corps de Lewy [27]. En neuroimagerie on retrouve souvent une atrophie très asymétrique des régions temporale, frontale inférieure et pariétale inférieure et des hypersignaux de la substance blanche [28].

Les DLFT-TDP de type A/B liées à une mutation C9orf72 sont également hétérogènes dans leur présentation mais il faut retenir la grande fréquence des présentation de DFTc-SLA, les mutations C9orf72 expliquant deux tiers des formes familiales de DFTc-SLA et jusqu'à 13\% des formes sporadiques [29]. Les présentations psychiatriques (10 à 40\%) sont particulières par leur survenue tardive et leur caractère atypique, prenant le masque d'une psychose avec hallucinations auditives, d'un trouble bipolaire ou d'un épisode dépressif majeur catatonique [30,31]. En neuroimagerie les DLFT-TDP A/B liées à une mutation C9orf72 se manifestent par une atrophie diffuse et symétrique des régions frontales, mais également temporopolaires, pariétales, occipitales, thalamiques et cérébelleuses [28]. De tels phénotypes anatomocliniques doivent inciter à proposer une analyse génétique si celle-ci n'a pas été proposée de façon systématique.

\section{Place du phénotype anatomoclinique}

C'est à ce stade, devant un phénotype de DFTc sans mutation identifiée et ne correspondant pas à une MA, que les corrélations clinicopathologiques sont plus incertaines. Une manière de procéder opérationnelle est de considérer l'âge, le phénotype clinique et la répartition de l'atrophie sur l'imagerie morphologique pour poser ses hypothèses étiologiques (figure 2) [8,32-34]. 


\section{Présentations cliniques spécifiques}

D'emblée, deux présentations cliniques de DFTc sont bien corrélées à la pathologie sousjacente. Les DFTc-SLA d'une part, qu'elles soient ou non causées par une mutation C9orf72, sont presque constamment liées à une pathologie DLFT-TDP de type B. Rarement les formes familiales de DFTc-SLA sont liées à une mutation sur les gènes TARDBP ou VCP (correspondant à une pathologie DLFT-TDP) ou à une mutation FUS (correspondant alors à une pathologie DLFT-FUS).

D'autre part, la mise en évidence d'emblée ou en cours de suivi d'un syndrome de PSP (paralysie supranucléaire du regard, hypertonie en extension, chutes en rétropulsion) associé à la DFTc est très spécifique de la PSP en tant qu'entité pathologique (DFLT-Tau 4R). Le syndrome cortico-basal est beaucoup moins spécifique car retrouvé dans la MA ou les mutations GRN. En outre le phénotype clinique emblématique des PSP et DCB n'est pas toujours complet ni présent. Le syndrome comportemental est le plus souvent modéré et l'atrophie frontotemporale modérée ou absente. Une atrophie mésencéphalique et un hypométabolisme préfrontal mésial/cingulaire électif peuvent contribuer au diagnostic de PSP [35] ; une atrophie frontale dorsale médiale et latérale asymétrique ou symétrique à celui de DCB [36] (figure 3d).

\section{DFTc « emblématiques »}

Deux étiologies se distinguent par leur phénotype DFTc «emblématiques », conjuguant un phénotype comportemental complet (remplissant précocement les 5 critères comportementaux de Rascovsky) à une atrophie frontotemporale sévère. Il s'agit de la maladie de Pick (DLFTTau 3R) et des DLFT-FUS. Les DLFT-FUS se distinguent souvent par un début très précoce (<45-50 ans) et la rapidité d'évolution de la maladie ; le comportement d'exploration orale (pica) est plus fréquent que dans les autres DLFT [8,37]. L'atrophie est symétrique, frontale ventromédiale et temporopolaire mais également du noyau caudé dans les FTLD-FUS ; elle est asymétrique, fronto-insulo-temporale dans la maladie de Pick [38]. C'est dans la maladie de Pick qu'on décrit classiquement l'atrophie sévère «en lame de couteau » du lobe frontal (figure 3a).

\section{DFTc «temporales »}

Certaines DFTc se distinguent par la prédominance temporale de l'atrophie et par un syndrome comportemental particulier avec une prédominance de la perte d'empathie et des 
compulsions et stéréotypies [32]. Ces «variants temporaux » peuvent représenter jusqu'à un tiers des cas de DFTc.

Lorsque l'atrophie est temporopolaire et très asymétrique, il s'agit le plus souvent d'une DLFT-TDP de type C. L'atrophie est le plus souvent à droite (figure 3b), car les DLFT-TDP de type $\mathrm{C}$ gauches sont le plus souvent révélées par un tableau classique d'APPs (démence sémantique). Le syndrome comportemental de ces formes temporales droites est particulier, marqué par une perte des convenances sociales, des traits obsessionnels, plus rarement des hallucinations visuelles complexes, des synesthésies ou une hyper-religiosité [39].

Une atrophie symétrique portant sur les régions temporales médiales et polaires doit faire évoquer une mutation MAPT chez un sujet jeune [32,40]. Chez les sujets âgés (>65 ans), le diagnostic de maladie à grains argyrophiles (appartenant au groupe des DLFT-Tau 4R) peut être évoqué [34]. L'atrophie est fronto-temporale ou diffuse (figure 3c). Les troubles de la mémoire épisodique sont constants [41,42] et le diagnostic de MA, souvent évoqué initialement, est remis en cause par les résultats des biomarqueurs ou l'évolution lente et atypique. Parmi ces atypies, les troubles du comportement, quoique modérés, remplissent souvent les critères de DFTc en associant changement de personnalité, perte des convenances, modification des goûts alimentaires et apathie.

\section{Autres présentations}

Les sous-types pathologiques restants sont les plus difficiles à caractériser. Les DLFT-TDP de type $\mathrm{A}$ et $\mathrm{B}$ sporadiques, quand elles ne sont pas liées à une mutation GRN ou C9orf72 et qu'elles ne sont pas associées à une SLA, manquent de caractère distinctif clinique ou radiologique. L'atrophie frontotemporale est moins marquée que dans la maladie de Pick et les DLFT-FUS. A l'instar des formes génétiques liées à une mutation GRN, Les DLFT-TDP de type A semblent présenter une atrophie diffuse, s'étendant aux régions temporales postérieures et pariétales [32,36] (figure 3e).

$\mathrm{Au}$ terme de cet exercice de corrélation entre phénotype clinique et radiologique et pathologie, l'un des aspects marquants est l'absence de marqueurs de classe moléculaire des DLFT, qui permettraient par exemple de distinguer une DLFT-Tau d'une DLFT-TDP. Ainsi la présentation clinique d'une DLFT-TDP de type A est souvent plus proche de certains soustypes de DLFT-Tau que des autres sous-types de DLFT-TDP. Certains marqueurs cliniques comme le syndrome parkinsonien, qui évoquerait volontiers une DLFT-Tau 4R, sont des «faux-amis » car retrouvé de façon assez uniforme dans l'ensemble des DLFT [43]. Ainsi même s'il existe une variabilité phénotypique importante, les corrélations clinico- 
pathologiques ne sont fiables qu'au sein de chaque sous-type histologique ou génétique. Nous proposons un arbre diagnostique pour porter un diagnostic de probabilité (figure 2).

\section{Conclusion}

Le diagnostic étiologique des DFTc reste une gageure en 2019. Néanmoins, les progrès de la génétique et la publication de grandes séries clinico-pathologiques multicentriques permettent de préciser les corrélations et d'affiner un diagnostic qui restera probabiliste en l'absence de mutation identifiée.

Distinguer une DFTc liée à une DLFT d'une présentation exécutive/comportementale de MA ou d'un trouble psychiatrique a une implication majeure pour la prise en charge thérapeutique et le pronostic. L'utilisation stricte des critères diagnostiques du DSM5 doit être encouragée pour distinguer trouble psychiatrique et DFTc. La réserve principale à formuler sur les critères actuels de DFTc concerne l'historique « relative préservation de la mémoire épisodique » qui doit être considérée avec circonspection, notamment dans le cadre du diagnostic différentiel avec une MA. Les révisions futures de ces critères verront sans doute disparaître la notion de préservation relative de la mémoire épisodique et encourageront probablement à l'évaluation de la cognition sociale. Le recours aux biomarqueurs du liquide cérébrospinal permet, en cas de négativité, d'écarter le diagnostic de MA ; mais l'interprétation des résultats doit rester prudente en cas de positivité car certains biomarqueurs de MA sont parfois positifs dans les DLFT et la probabilité d'une association de pathologies augmente avec l'âge.

Le diagnostic étiologique des DFTc est loin d'être uniquement un jeu intellectuel, même en l'absence de traitement curatif. A la variabilité des lésions pathologiques sous-jacentes répond une variabilité pronostique ; ainsi le pronostic vital et fonctionnel est radicalement différent entre une DLFT-FUS ou une DLFT-TDP de type B associée à une SLA, dont le pronostic est très sévère, et une DLFT-TDP de type $\mathrm{C}$ ou une maladie à grains argyrophiles, dont l'évolution est particulièrement lente. La rareté des essais thérapeutiques de traitements disease modifyer dans les DFTc non génétiques tient à l'absence de marqueur fiable de la pathologie sous-jacente, car des traitements comme les immunothérapies anti-Tau sont d'ores et déjà disponibles et testés dans d'autres taupathies que les DLFT-Tau.

Les progrès dans les corrélations clinico-pathologiques dépendent des dons de cerveau. Il ne faut cesser de promouvoir son importance (consulter le site Neuro-CEB www.neuroceb.org pour plus d'informations). L'avenir proche verra sans doute l'apparition de marqueurs en neuroimagerie ou en biochimie. Parmi ces derniers, le dosage de la chaîne légère des neurofilaments dans le sang ou dans le liquide cérébrospinal semble particulièrement prometteur pour le diagnostic différentiel entre DLFT et troubles psychiatriques et pour le 
pronostic [44]. L'avènement de radiotraceurs de taupathie capables d'identifier les DLFT-Tau par rapport aux autres est également un espoir [12]. Malgré ces avancées attendues, la clinique restera indispensable pour guider l'utilisation de ces biomarqueurs parfois coûteux, identifier les sous-types au sein des classes moléculaires de DLFT, conforter les corrélations clinico-pathologiques et repérer les discordances, notamment dans les cas d'associations pathologiques.

\section{Liens d'intérêts}

Les auteurs déclarent n'avoir aucun lien d'intérêts en relation avec cet article. 


\section{Points essentiels}

- Le diagnostic positif et étiologique du variant comportemental de dégénérescence lobaire frontotemporale (DFTc) reste un enjeu.

- La préservation relative de la mémoire épisodique décrite dans les critères actuels concerne moins de la moitié des DFTc.

- L'utilisation stricte du DSM5 et l'analyse qualitative et quantitative des troubles du comportement sont la clef du diagnostic différentiel entre DFTc et trouble psychiatrique.

- La recherche de mutations GRN et C9orf72 peut être proposée systématiquement, y compris dans les formes sporadiques de DFTc.

- Les DFTc associées à une pathologie motoneuronale sont souvent liées à une DLFT-TDP de type B.

- Les DFTc avec une atrophie à prédominance temporale peuvent être liées à une mutation MAPT, à une pathologie DLFT-TDP type C ou à une maladie à grains argyrophiles.

- Les DFTc sévères et caricaturales cliniquement et en imagerie sont souvent liées à une DLFT-FUS chez les sujets très jeunes ou à une DLFT-Tau 3R (maladie de Pick) chez les sujets plus âgés. 


\section{Références}

[1] McKhann GM, Knopman DS, Chertkow H, Hyman BT, Jack CR Jr, Kawas CH, et al. The diagnosis of dementia due to Alzheimer's disease: recommendations from the National Institute on Aging-Alzheimer's Association workgroups on diagnostic guidelines for Alzheimer's disease. Alzheimers Dement, vol. 7, 2011, pp. 263-9. doi:10.1016/j.jalz.2011.03.005.

[2] McKeith IG, Boeve BF, Dickson DW, Halliday G, Taylor J-P, Weintraub D, et al. Diagnosis and management of dementia with Lewy bodies: Fourth consensus report of the DLB Consortium. Neurology 2017;89:88-100. doi:10.1212/WNL.0000000000004058.

[3] Sachdev P, Kalaria R, O'Brien J, Skoog I, Alladi S, Black SE, et al. Diagnostic criteria for vascular cognitive disorders: a VASCOG statement. Alzheimer Dis Assoc Disord 2014;28:206-18. doi:10.1097/WAD.0000000000000034.

[4] Höglinger GU, Respondek G, Stamelou M, Kurz C, Josephs KA, Lang AE, et al. Clinical diagnosis of progressive supranuclear palsy: The movement disorder society criteria. Mov Disord 2017;32:853-64. doi:10.1002/mds.26987.

[5] Postuma RB, Berg D, Stern M, Poewe W, Olanow CW, Oertel W, et al. MDS clinical diagnostic criteria for Parkinson's disease. Mov Disord 2015;30:1591-601. doi:10.1002/mds.26424.

[6] Gorno-Tempini ML, Hillis AE, Weintraub S, Kertesz A, Mendez M, Cappa SF, et al. Classification of primary progressive aphasia and its variants. Neurology, vol. 76, 2011, pp. 1006-14. doi:10.1212/WNL.0b013e31821103e6.

[7] Bergeron D, Gorno-Tempini ML, Rabinovici GD, Santos-Santos MA, Seeley W, Miller BL, et al. Prevalence of amyloid- $\beta$ pathology in distinct variants of primary progressive aphasia. Ann Neurol 2018;84:729-40. doi:10.1002/ana.25333.

[8] Perry DC, Brown JA, Possin KL, Datta S, Trujillo A, Radke A, et al. Clinicopathological correlations in behavioural variant frontotemporal dementia. Brain 2017;140:3329-45. doi:10.1093/brain/awx254.

[9] Pasquier F, Lebouvier T, Lebert F. Frontotemporal Dementia. Neuropsychiatric Symptoms of Cognitive Impairment and Dementia, vol. 51, Cham: Springer International Publishing; 2016, pp. 279-302. doi:10.1007/978-3-319-39138-0_13.

[10] Bang J, Spina S, Miller BL. Frontotemporal dementia. Lancet 2015;386:1672-82. doi:10.1016/S0140-6736(15)00461-4.

[11] Lashley T, Rohrer JD, Mead S, Revesz T. Review: an update on clinical, genetic and pathological aspects of frontotemporal lobar degenerations. Neuropathol Appl Neurobiol 2015;41:858-81. doi:10.1111/nan.12250.

[12] Lebouvier T, Pasquier F, Buée L. Update on tauopathies. Current Opinion in Neurology 2017;30:589-98. doi:10.1097/WCO.0000000000000502.

[13] Le Ber I. Genetics of frontotemporal lobar degeneration: an up-date and diagnosis algorithm. Rev Neurol (Paris) 2013;169:811-9. doi:10.1016/j.neurol.2013.07.014.

[14] Rascovsky K, Hodges JR, Knopman D, Mendez MF, Kramer JH, Neuhaus J, et al. Sensitivity of revised diagnostic criteria for the behavioural variant of frontotemporal dementia. Brain 2011;134:2456-77. doi:10.1093/brain/awr179.

[15] Frontotemporal behavioral scale. Alzheimer Dis Assoc Disord 1998;12:335-9.

[16] Boutoleau-Bretonnière C, Evrard C, Hardouin JB, Rocher L, Charriau T, EtcharryBouyx F, et al. DAPHNE: A New Tool for the Assessment of the Behavioral Variant of Frontotemporal Dementia. Dement Geriatr Cogn Dis Extra 2015;5:503-16. doi:10.1159/000440859.

[17] Godefroy O, Azouvi P, Robert P, Roussel M, LeGall D, Meulemans T, et al. Dysexecutive syndrome: Diagnostic criteria and validation study. Ann Neurol 2010;68:855-64. doi:10.1002/ana.22117. 
[18] Hornberger M, Piguet O. Episodic memory in frontotemporal dementia: a critical review. Brain 2012;135:678-92. doi:10.1093/brain/aws011.

[19] Bertoux M, de Souza LC, Corlier F, Lamari F, Bottlaender M, Dubois B, et al. Two distinct amnesic profiles in behavioral variant frontotemporal dementia. Biol Psychiatry 2014;75:582-8. doi:10.1016/j.biopsych.2013.08.017.

[20] Bertoux M, de Souza LC, O’Callaghan C, Greve A, Sarazin M, Dubois B, et al. Social Cognition Deficits: The Key to Discriminate Behavioral Variant Frontotemporal Dementia from Alzheimer's Disease Regardless of Amnesia? J Alzheimers Dis 2016;49:1065-74. doi:10.3233/JAD-150686.

[21] Ossenkoppele R, Pijnenburg YAL, Perry DC, Cohn-Sheehy BI, Scheltens NME, Vogel JW, et al. The behavioural/dysexecutive variant of Alzheimer's disease: clinical, neuroimaging and pathological features. Brain 2015;138:2732-49. doi:10.1093/brain/awv191.

[22] Ducharme S, Price BH, Larvie M, Dougherty DD, Dickerson BC. Clinical Approach to the Differential Diagnosis Between Behavioral Variant Frontotemporal Dementia and Primary Psychiatric Disorders. Am J Psychiatry 2015;172:827-37. doi:10.1176/appi.ajp.2015.14101248.

[23] Haroche A, Amad A, Fovet T, Geoffroy PA, Defebvre L. Les maladies neurologiques à expression psychiatrique : importance de l'examen neurologique. Annales MédicoPsychologiques, Revue Psychiatrique 2017;175:462-8.

doi:10.1016/j.amp.2017.03.004.

[24] Reus LM, Vijverberg EG, Tijms BM, Kate MT, Gossink F, Krudop WA, et al. Disease trajectories in behavioural variant frontotemporal dementia, primary psychiatric and other neurodegenerative disorders presenting with behavioural change. J Psychiatr Res 2018;104:183-91. doi:10.1016/j.jpsychires.2018.07.014.

[25] Devenney E, Forrest SL, Xuereb J, Kril JJ, Hodges JR. The bvFTD phenocopy syndrome: a clinicopathological report. Journal of Neurology, Neurosurgery \& Psychiatry 2016;87:1155-6. doi:10.1136/jnnp-2015-312826.

[26] Gossink FT, Dols A, Kerssens CJ, Krudop WA, Kerklaan BJ, Scheltens P, et al. Psychiatric diagnoses underlying the phenocopy syndrome of behavioural variant frontotemporal dementia. Journal of Neurology, Neurosurgery \& Psychiatry 2016;87:64-8. doi:10.1136/jnnp-2014-308284.

[27] Rademakers R, Baker M, Gass J, Adamson J, Huey ED, Momeni P, et al. Phenotypic variability associated with progranulin haploinsufficiency in patients with the common 1477C--> T (Arg493X) mutation: an international initiative. The Lancet Neurology 2007;6:857-68. doi:10.1016/S1474-4422(07)70221-1.

[28] Whitwell JL, Weigand SD, Boeve BF, Senjem ML, Gunter JL, DeJesus-Hernandez $\mathrm{M}$, et al. Neuroimaging signatures of frontotemporal dementia genetics: C9ORF72, tau, progranulin and sporadics. Brain 2012;135:794-806. doi:10.1093/brain/aws001.

[29] Le Ber I, Camuzat A, Guillot-Noel L, Hannequin D, Lacomblez L, Golfier V, et al. C9ORF72 repeat expansions in the frontotemporal dementias spectrum of diseases: a flow-chart for genetic testing. J Alzheimers Dis 2013;34:485-99. doi:10.3233/JAD121456.

[30] Le Ber I. Frontotemporal lobar dementia and amyotrophic lateral sclerosis associated with c9orf72 expansion. Rev Neurol (Paris) 2015;171:475-81. doi:10.1016/j.neurol.2015.04.004.

[31] Ducharme S, Bajestan S, Dickerson BC, Voon V. Psychiatric Presentations of C9orf72 Mutation: What Are the Diagnostic Implications for Clinicians? J

Neuropsychiatry Clin Neurosci 2017;29:195-205. doi:10.1176/appi.neuropsych.16090168.

[32] Rohrer JD, Lashley T, Schott JM, Warren JE, Mead S, Isaacs AM, et al. Clinical and neuroanatomical signatures of tissue pathology in frontotemporal lobar degeneration. Brain 2011;134:2565-81. doi:10.1093/brain/awr198. 
[33] Whitwell JL, Josephs KA. Neuroimaging in frontotemporal lobar degeneration-predicting molecular pathology. Nat Rev Neurol 2012;8:131-42.

doi:10.1038/nrneurol.2012.7.

[34] Seo SW, Thibodeau M-P, Perry DC, Hua A, Sidhu M, Sible I, et al. Early vs late age at onset frontotemporal dementia and frontotemporal lobar degeneration. Neurology 2018;90:e1047-56. doi:10.1212/WNL.0000000000005163.

[35] Ge J, Wu J, Peng S, Wu P, Wang J, Zhang H, et al. Reproducible network and regional topographies of abnormal glucose metabolism associated with progressive supranuclear palsy: Multivariate and univariate analyses in American and Chinese patient cohorts. Hum Brain Mapp 2018;39:2842-58. doi:10.1002/hbm.24044.

[36] Whitwell JL, Jack CR, Parisi JE, Knopman DS, Boeve BF, Petersen RC, et al. Imaging signatures of molecular pathology in behavioral variant frontotemporal dementia. J Mol Neurosci 2011;45:372-8. doi:10.1007/s12031-011-9533-3.

[37] Snowden JS, Hu Q, Rollinson S, Halliwell N, Robinson A, Davidson YS, et al. The most common type of FTLD-FUS (aFTLD-U) is associated with a distinct clinical form of frontotemporal dementia but is not related to mutations in the FUS gene. Acta Neuropathol 2011;122:99-110. doi:10.1007/s00401-011-0816-0.

[38] Gordon E, Rohrer JD, Fox NC. Advances in neuroimaging in frontotemporal dementia. J Neurochem 2016;138 Suppl 1:193-210. doi:10.1111/jnc.13656.

[39] Chan D, Anderson V, Pijnenburg Y, Whitwell J, Barnes J, Scahill R, et al. The clinical profile of right temporal lobe atrophy. Brain 2009;132:1287-98. doi:10.1093/brain/awp037.

[40] Fumagalli GG, Basilico P, Arighi A, Bocchetta M, Dick KM, Cash DM, et al. Distinct patterns of brain atrophy in Genetic Frontotemporal Dementia Initiative (GENFI) cohort revealed by visual rating scales. Alzheimers Res Ther 2018;10:46. doi:10.1186/s13195-018-0376-9.

[41] Ferrer I, Santpere G, van Leeuwen FW. Argyrophilic grain disease. Brain 2008;131:1416-32. doi:10.1093/brain/awm305.

[42] Argyrophilic Grain Disease: Demographics, Clinical, and Neuropathological Features From a Large Autopsy Study. J Neuropathol Exp Neurol 2016;75:628-35. doi:10.1093/jnen/nlw034.

[43] Baizabal-Carvallo JF, Jankovic J. Parkinsonism, movement disorders and genetics in frontotemporal dementia. Nat Rev Neurol 2016;12:175-85. doi:10.1038/nrneurol.2016.14.

[44] Zetterberg H, van Swieten JC, Boxer AL, Rohrer JD. Fluid biomarkers for frontotemporal dementias. Neuropathol Appl Neurobiol 2018;148:409. doi:10.1111/nan.12530. 


\section{Figures}

Figure 1 : Neuropathologie et génétique des DLFT. DNF : dégénérescences neurofibrillaires ; INC : inclusions neuronales cytoplasmiques ; ND : neurones dystrophiques ; IIN inclusions intranucléaires neuronales

\begin{tabular}{|c|c|c|c|c|}
\hline \multicolumn{2}{|c|}{$\begin{array}{l}\text { Groupe } \\
\text { moléculaire }\end{array}$} & \multicolumn{2}{|c|}{ Sous-type histologique } & \multirow[t]{2}{*}{$\begin{array}{l}\text { Mutation } \\
\text { possible }\end{array}$} \\
\hline \multirow{7}{*}{\multicolumn{2}{|c|}{$\begin{array}{c}\text { DLFT-Tau } \\
\sim 40 \%\end{array}$}} & Taupathie $3 R$ & Maladie de Pick & \\
\hline & & Corps de Pick & DLFT-Tau 3R par mutation MAPT & MAPT \\
\hline & & Taunathie $\mathbf{A R}$ & PSP / DCB & \\
\hline & & $\begin{array}{l}\text { Inclusions } \\
\text { neurogliales de }\end{array}$ & Maladie à grains argyrophiles & \\
\hline & & proteine rau & DLFT-Tau 4 R par mutation MAPT & MAPT \\
\hline & & Taupathie 3/4R & Démence avec DNF & \\
\hline & & Divi & DLFT-Tau 3/4R par mutation MAPT & MAPT \\
\hline \multirow{8}{*}{ 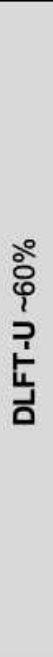 } & \multirow{4}{*}{ 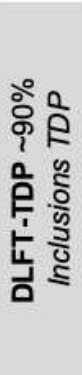 } & $\begin{array}{l}\text { Type A } \\
\text { INC, ND } \pm I I N\end{array}$ & DLFT-TDP type A & $\begin{array}{l}\text { GRN } \\
\text { C9orf72 }\end{array}$ \\
\hline & & $\begin{array}{l}\text { Type B } \\
I N C \pm N D\end{array}$ & DLFT-TDP type B & C9orf72 \\
\hline & & $\begin{array}{l}\text { Type C } \\
\text { ND }\end{array}$ & DLFT-TDP type C & \\
\hline & & $\begin{array}{l}\text { Type D } \\
I I N, N D \pm I N C\end{array}$ & DLFT-TDP type B & VCP \\
\hline & \multirow{3}{*}{$\frac{3}{\frac{3}{6}} \frac{8}{8}$} & \multicolumn{2}{|c|}{ DLFT atypique avec inclusions neuronales à ubiquitine } & \\
\hline & & \multicolumn{2}{|c|}{ Maladie des inclusions neuronales de filaments intermédiaires } & \\
\hline & & \multicolumn{2}{|c|}{ Maladie des corps d'inclusions basophiles } & \\
\hline & DAFT & \multicolumn{2}{|c|}{ DLFT avec inclusions ubiquitinylées } & CHMP2B \\
\hline \multicolumn{2}{|c|}{ Autre } & \multicolumn{2}{|c|}{ Démance sans histologie spécifique } & \\
\hline
\end{tabular}



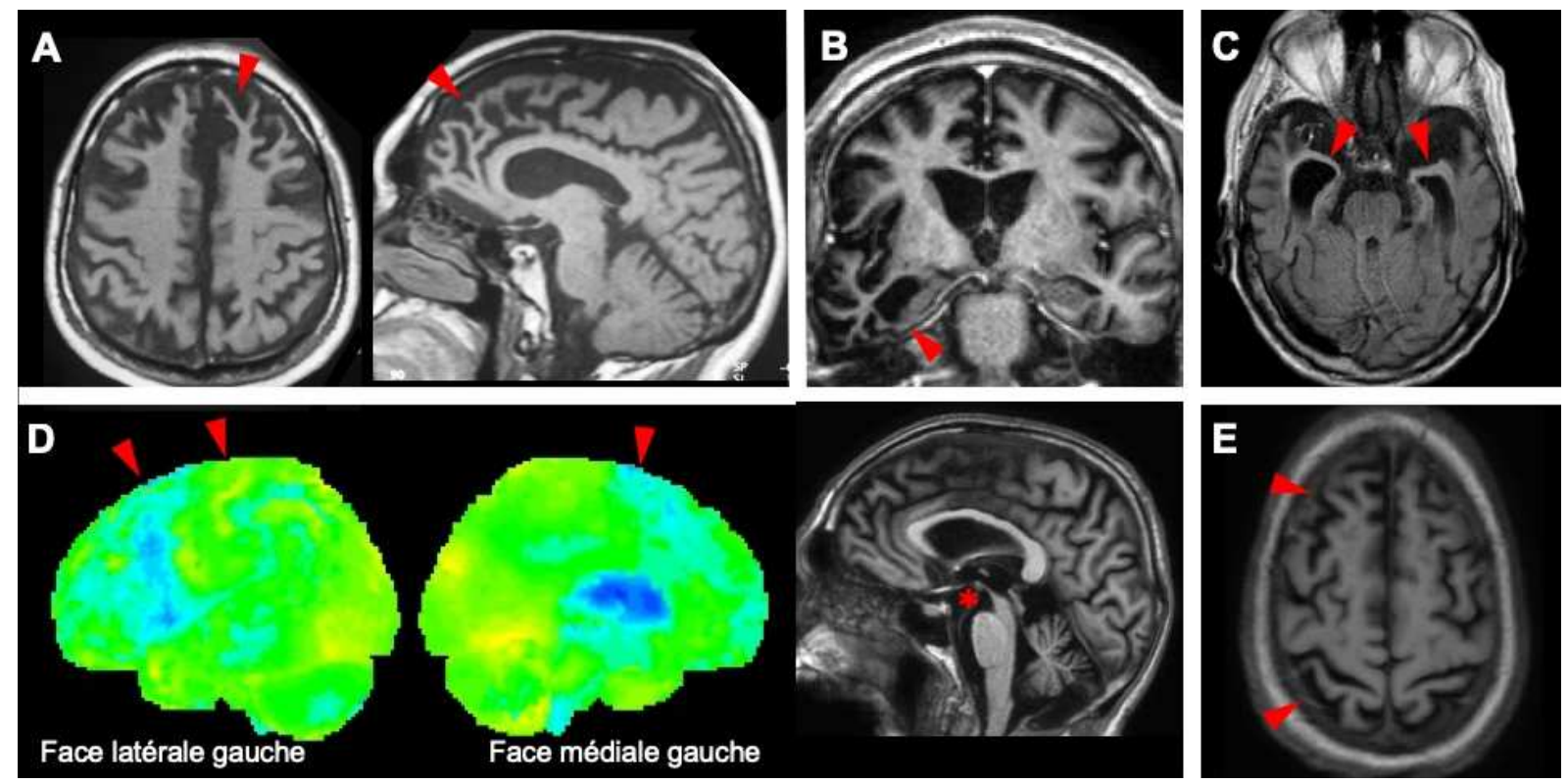

Figure 2 : Corrélations entre neuroimagerie et pathologie. A. Homme de 55 ans présentant une DFTc sévère depuis l'âge de 50 ans ; l'analyse histologique au décès à l'âge de 69 ans montre une maladie de Pick. L'atrophie «en lame de couteau» du cortex frontal, ici légèrement asymétrique aux dépens de la gauche, est présente dès le début de la maladie (têtes de flèche). B. Homme de 64 ans présentant une DFTc avec stéréotypies, rigidité du comportement et perte des convenances ; l'analyse histologique au décès à l'âge de 72 ans montre une DLFT-TDP type $\mathrm{C}$ à prédominance droite. L'IRM objective une atrophie temporale majeure très asymétrique aux dépens de la droite (têtes de flèche). C. Femme de 89 ans présentant une amnésie majeure et un syndrome comportemental modéré remplissant les critères de DFTc ; l'IRM objective une atrophie temporopolaire et temporale mésiale majeure, largement prédominante (têtes de flèche). A son décès à 91 ans, l'analyse histologique montre une maladie à grains argyrophiles associée à des lésions vasculaires au second plan. D. Patient de 65 ans présentant une DFTc à prédominance apathique et des troubles du langage (diminution de la fluence, anomie) au second plan. L'examen n'objective pas de syndrome de paralysie supranucléaire progressive ni de syndrome corticobasal. Le diagnostic de DLFT-Tau 4R a été évoqué sur un hypométabolisme des régions dorso-frontales et centrales à prédominance gauche sur la TEP-FDG (têtes de flèche), compatible avec une DCB, et sur une atrophie mésencéphalique (astérisque) 
compatible avec une PSP. Il a été confirmé à l'autopsie. e. Patient de 55 ans présentant une atrophie modérée diffuse sans nette prédilection frontale, incluant une atrophie pariétale (têtes de flèche), en rapport avec une DLFT-TDP de type A d'origine génétique (mutation GRN). 
Figure 3 : Arbre diagnostique

Syndrome comportemental remplissant les critères de DFTc selon Rascovsky (2011)

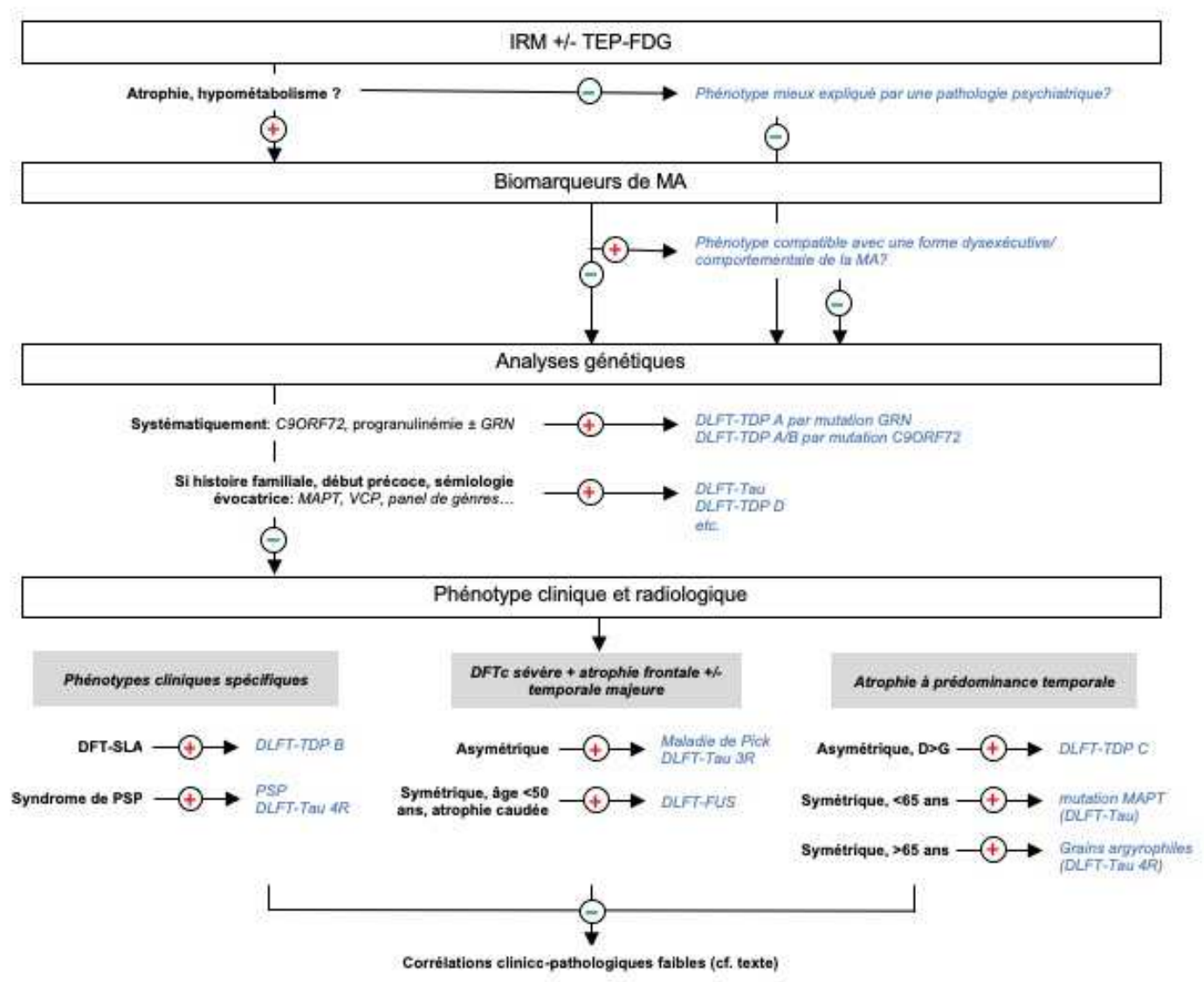


Tableau 1 - Critères diagnostiques de DFTc (D’après Rascovsky et al., 2011). Note : précoce se réfère à la survenue des symptômes dans les 3 premières années.

1. Pathologie neurodégénérative : Le critère suivant doit être présent.

A. Détérioration progressive du comportement et/ou de la cognition sur la base du suivi ou de l'interrogatoire (rapport d'un informant fiable)

2. Variant comportemental de DFT possible: Trois des symptômes comportementaux/cognitifs $(A-F)$ doivent être présents. Ces symptômes doivent être persistants ou récurrents, plutôt qu'isolés ou rares.

A. Désinhibition comportementale précoce *

- Comportement social inapproprié

- Perte des convenances ou de politesse

- Actes impulsifs, irréfléchis, voire imprudents

B. Apathie/Inertie précoce *

- Apathie

- Inertie

C. Perte de sympathie ou d'empathie précoce *

- Réponse diminuée aux besoins et sentiments des autres personnes

- Baisse de l'intérêt social, altération des relations interpersonnelles, émoussement affectif

D. Comportement persévératif, stéréotypé ou compulsif/obsessionnel précoce *

- Mouvements répétitifs simples

- Comportements complexes compulsifs ou ritualisés

- Stéréotypies verbales

E. Hyperoralité et changement des habitudes alimentaires *

- Modifications des préférences alimentaires

- Gloutonnerie, consommation aggravée d'alcool ou de cigarettes

- Hyperoralité (exploration orale, consommation d'objets non comestibles)

F. Profil neuropsychologique (tous les symptômes suivants doivent être présents)

- Déficit dans les tests exécutifs

- Préservation relative de la mémoire épisodique

- Préservation relative des fonctions visuospatiales

* Un des symptômes suivants doit être présent

3. Variant comportemental de DFT probable: Tous les critères suivants doivent être présents.

A. Remplit les critères de variant comportemental de DFT possible

B. Présence d'un déclin fonctionnel significatif (rapport de l'informant ou sur la base d'échelles cliniques de syndrome démentiel ou d'activités de vie quotidienne)

C. Résultats d'imagerie compatibles avec le diagnostic de variant comportemental de DFT (un des critères suivants soit être présent)

- Atrophie frontale et/ou temporale antérieure en IRM ou Scanner

- Hypoperfusion ou hypométabolisme frontal et/ou temporal antérieur en SPECT ou TEP

4. Variant comportemental de DFT avec pathologie de DLFT définie : Le critère $A$ et soit le critère $B$ ou $C$ doivent être présents. 


\begin{tabular}{|l|l|}
\hline & $\begin{array}{l}\text { A. Remplit les critères de variant comportemental de DFT possible ou probable } \\
\text { B. Preuve histopathologique de DLFT par biopsie cérébrale ou examen post } \\
\text { mortem } \\
\text { C. Présence d'une mutation génétique pathogène. }\end{array}$ \\
\hline $\begin{array}{l}\text { 5. Variant comportemental de DFT exclu : Les critères A et B doivent être négatifs pour } \\
\text { tout diagnostic de variant comportemental de DFT. Le critères C peut être positif dans le } \\
\text { cadre d'un diagnostic de variant comportemental de DFT possible, mais doit être négatif } \\
\text { dans le cadre d'un diagnostic de variant comportemental de DFT probable. }\end{array}$ \\
\hline $\begin{array}{l}\text { A. Le profil des troubles est mieux expliqué par une autre pathologie non } \\
\text { neurodégénérative du système nerveux ou une autre affection médicale. } \\
\text { B. Les altérations comportementales sont mieux expliquées par un diagnostic } \\
\text { psychiatrique } \\
\text { C. Biomarqueurs fortement indicatifs de maladie d'Alzheimer ou d'autres } \\
\text { pathologies neurodégénératives. }\end{array}$ \\
\hline
\end{tabular}

